\title{
PROGRESSO TEMPORAL DA FERRUGEM ALARANJADA EM CULTIVARES DE CAFEEIRO NO OESTE DE SÃO PAULO
}

\author{
E.M. Paulo ${ }^{1}$, S.M.N.M. Montes ${ }^{1}$, I.H. Fischer ${ }^{2}$
}

${ }^{1}$ Polo Regional de Desenvolvimento de Tecnologia dos Agronegócios da Alta Sorocabana, Rod. Raposo Tavares, km 561, CEP 19015-970, Presidente Prudente, SP, Brasil. E-mail: edisonpaulo@apta.sp.gov.br

\section{RESUMO}

A ferrugem é a doença mais comum das lavouras de café. O trabalho estudou nos cultivares de Coffea arabica L. Acaiá IAC 474-19, Catuaí Amarelo IAC 47, Icatu Amarelo IAC 2944, Icatu Vermelho IAC 4045, Obatã IAC 1669-20 e Apoatã IAC 2258 (Coffea canephora Pierre ex Fhroen) a incidência da ferrugem. O ensaio foi realizado em Adamantina, SP, onde os cafeeiros foram plantados no espaçamento de $4,0 \times 2,0 \mathrm{~m}$, com duas plantas por cova e dispostos em 6 linhas com 15 a 20 covas cada. No período entre junho de 2008 a dezembro de 2009, quinzenalmente, coletou-se ao acaso 30 folhas obtidas nos terços superior, médio e inferior dos cafeeiros e calculou-se a porcentagem das folhas com sintomas característicos da ferrugem. Calculou-se a área abaixo da curva de progresso da ferrugem para cada um dos cultivares. Constatou-se a doença na Icatu Amarelo IAC 2944 e Icatu Vermelho IAC 4045, cultivares descritos como resistentes/tolerantes à ferrugem. Concluiuse que a incidência diferiu entre os cultivares e diminuiu com o aumento da temperatura, mas não com a precipitação pluvial. A incidência da doença menor que $5 \%$ nos cafeeiros ocorreu com temperaturas máximas médias mensais maiores que $30^{\circ} \mathrm{C}$.

PALAVRAS-CHAVE: Coffea arabica L., Hemileia vastatrix, doença, resistência, clima.

\section{ABSTRACT}

RUST IS THE MOST COMMON DISEASE IN THE COFFEE PLANTATIONS. This work aimed to evaluate the incidence of coffee rust in the following cultivars of Coffea arabica L.: Acaiá IAC 474-19, Catuaí Amarelo IAC 47, Icatu Amarelo IAC 2944, Icatu Vermelho IAC 4045, Obatã IAC 1669-20 and Apoatã IAC 2258 (Coffea canephora Pierre ex Fhroen). The experiment was conducted in Adamantina, northwest region of the state of São Paulo, Brazil. The coffee trees were planted at a spacing of $4.0 \times 2.0 \mathrm{~m}$ with two plants per hole. Each plot consisted of six lines with 15 to 20 holes each. From June 2008 to December 2009, 30 leaves were randomly collected from the upper, middle and bottom portions of the trees. The leaf rust was found in all cultivars, including on Catuai Amarelo IAC 2944 and Vermelho IAC 4045, although both cultivars are considered resistant to the disease in field conditions. The infections differed significantly among cultivars. The rust symptoms declined with increasing temperature, and the rainfall did not affect the incidence of disease. The less-than-5\% infection rate coincided with the occurrence of maximum monthly mean temperatures greater than $30^{\circ} \mathrm{C}$.

KEY WORDS: Coffea arabica L., Hemileia vastatrix, disease resistance, climate.

\section{INTRODUÇÃO}

A ferrugem (Hemileiavastatrix Berkeley \& Broome) do cafeeiro (Coffea arabica L.) induz a desfolha por ocasião da colheita, a queda precoce das folhas, o menor vingamento da florada e dos frutos na fase de chumbinho e a seca dos ramos plagiotrópicos das plantas de café, cujo conjunto de eventos pode reduzir em mais de $50 \%$ a produção das lavouras (ZAMBolim et al., 1997).
Os uredósporos da ferrugem são disseminados pelo vento e pela água e a germinação (ZAMBOLIM et al., 2005) e a longevidade do esporo (RibeIRo et al., 1978) são dependentes da temperatura do ar. A germinação ótima dos uredósporos acontece quando há molhamento foliar (ZАмBoLIM et al., 2005).

A doença relaciona-se diretamente com onível de produção do cafeeiro (Miguel et al.,1977; ZAмвoLimet al.,2005) e é favorecida por deficiências nutricionais, manejo inadequado eespaçamentos reduzidos entre

${ }^{2}$ Polo Regional de Desenvolvimento de Tecnologia dos Agronegócios do Centro Oeste, Bauru, SP, Brasil. 
as linhas da cultura que causem aumento do autossombreamento das folhas (VIEIRA JunIOR et al., 2008).

Plantas que ainda não começaram a produzir e com menor porte em relação ao espaçamento e maior arejamento da copa têm microclima desfavorável à ocorrência da ferrugem (ZAMBolim et al., 2005), pelo que se observa o aumento da intensidade da doença após as primeiras produções do cafeeiro (Chalfoun, 1997).

Ocontrole da ferrugem pode ser feito por meio de fungicidas ou pelo emprego de métodos alternativos como o controle biológico. A indução de resistência nos cultivares é considerada o controle ideal da ferrugem, pois o uso contínuo de produtos químicos aumenta os custos de produção, impacta o ambiente e pode causar a resistência dos fitopatógenos.

Cultivares sem resistência à ferrugem são utilizados amplamente nos sistemas de produção de café e podem diferir quanto ao grau de resistência à $H$. vastatrix, enquanto as resistentes podem, ao longo do tempo, tornarem-se suscetíveis às novas raças do fungo originadas por mutações genéticas (FAzUOLI et al., 2007).

O monitoramento da incidência da ferrugem é importante porque permite conhecer as épocas críticas de ocorrência, a identificação dos níveis de dano econômico e o momento ideal de iniciar o controle químico da doença nos cafezais (VIEIRA JUNIOR et al., 2008), o que por vezes pode ser conseguido com uma única aplicação de fungicida em lavouras com baixa a média carga pendente (GARÇOM, 2004).

O trabalho estudou o progresso temporal e a incidência de cultivares de cafeeiro pela ferrugem alaranjada no clima Aw do Oeste de São Paulo.

\section{MATERIAL E MÉTODOS}

Oexperimento foi realizado no Município de Adamantina que tem clima tropical com verão chuvoso e inverno seco do tipo Aw, segundo Köeppen, com temperatura média do mês mais frio maior que $18^{\circ}$ C (TREMOCOLDI; BRUNINI, 2008). Os dados de temperatura e precipitação pluvial do período experimental estão na Tabela 1.

A ocorrência de pústulas infecciosas características da ferrugem foi avaliada nos cultivares Acaiá IAC 474-19, Catuaí Amarelo IAC 47, Icatu Amarelo IAC 2944, Icatu Vermelho IAC 4045, Obatã IAC 1669-20 e Apoatã IAC 2258 (Coffea canephora Pierre ex Fhroen), em pé franco com idade aproximada de 13 anos, plantados no espaçamento de $4,0 \times$ 2,0 m, com duas plantas por cova, dispostos em 6 linhas com 15 a 20 covas cada na mesma área experimental. No período entre junho de 2008 a dezembro de 2009, quinzenalmente, coletou-se ao acaso 30 folhas obtidas nos terços superior, médio e inferior dos cafeeiros de cada uma das quatro linhas centrais de cada cultivar. Separou-se as folhas coletadas em folhas com e sem sintomas característicos da ferrugem, quais sejam: manchas de coloração amarelo-pálida, pequenas, de 1 a 3 $\mathrm{mm}$ de diâmetro, que evoluem, atingindo até $2 \mathrm{~cm}$ de diâmetro, quando então apresentam aspecto pulverulento com produção de uredósporos de coloração amarelo-alaranjada, observados na face inferior das folhas e, na face superior, manchas cloróticas amareladas correspondentes aos limites da pústula na face inferior (ZАмBolim et al., 1997).

A incidência da ferrugem foi estimada pelo número de folhas com sintoma da doença dividido pelo número total de folhas da amostra, multiplicado por 100, assim expressa em porcentagem de incidência. Após a obtenção dos dados de incidência da ferrugem, calculou-se a área abaixo da curva de progresso da doença (AACPD), de acordo com a equação proposta por CAMPBELL; MADDEN (1990):

$$
\mathrm{AACPD}=\sum_{i}^{n-1}\left[\frac{Y i+Y i+1}{2}\right](t i+1-t i), \text { em que: }
$$

$\mathrm{y}_{\mathrm{i}}=$ proporção da doença na i-ésima observação;

$t_{i}=$ tempo em dias na i-ésima observação;

$\mathrm{n}$ = número total de observações; $\left(\mathrm{y}_{\mathrm{i}}{ }^{+} \mathrm{y}_{\mathrm{i}+1}\right)$ a altura média do retângulo entre os pontos $\mathrm{y}_{\mathrm{i}} \mathrm{ey}_{\mathrm{i}+1} ; \mathrm{e}\left(\mathrm{t}_{\mathrm{i}+1}-\mathrm{t}_{\mathrm{i}}\right) \mathrm{a}$ diferença da base do retângulo entre os pontos $t_{i+1}$ et $t_{i}$.

Os resultados de incidência de ferrugem do cafeeiro, expressos como AACPD, foram submetidos à análise de variância e as médias dos cultivares comparadas pelo teste de Tukey $(P<0,05)$.

Os dados deincidência de ferrugem noscultivares foram correlacionados com as temperaturas máximas e mínimas médias e com a precipitação pluvial acumulada de cada período de avaliação, obtidas na estação meteorológica automática do Polo Regional de Desenvolvimento Tecnológico dos Agronegócios da Alta Paulista, posteriormente analisados pelo teste $\mathrm{F}(P<0,05)$.

\section{RESULTADOS E DISCUSSÃO}

Os esporos da ferrugem do cafeeiro encontram condições ótimas para germinação quando há molhamento foliar prolongado e temperatura do ar de $22^{\circ} \mathrm{C}$ associadas à alta densidade foliar (ZAMBolim et al., 2005). O processo de germinação desses uredósporos é favorecido pela presença da água na forma líquida, mas ocorre também sob ar úmido em 24 horas e temperatura de $24^{\circ} \mathrm{C}$ (GALLI; CARVALHO, 1980).

As temperaturas médias do ar do período experimental situaram-se entre $15^{\circ} \mathrm{C}$ e $28,5^{\circ} \mathrm{C}$ (Tabela 1 ), condição térmica que não causa inibição da germinação dos uredósporos da ferrugem do cafeeiro (ZAMBOLIM et al., 2005). 


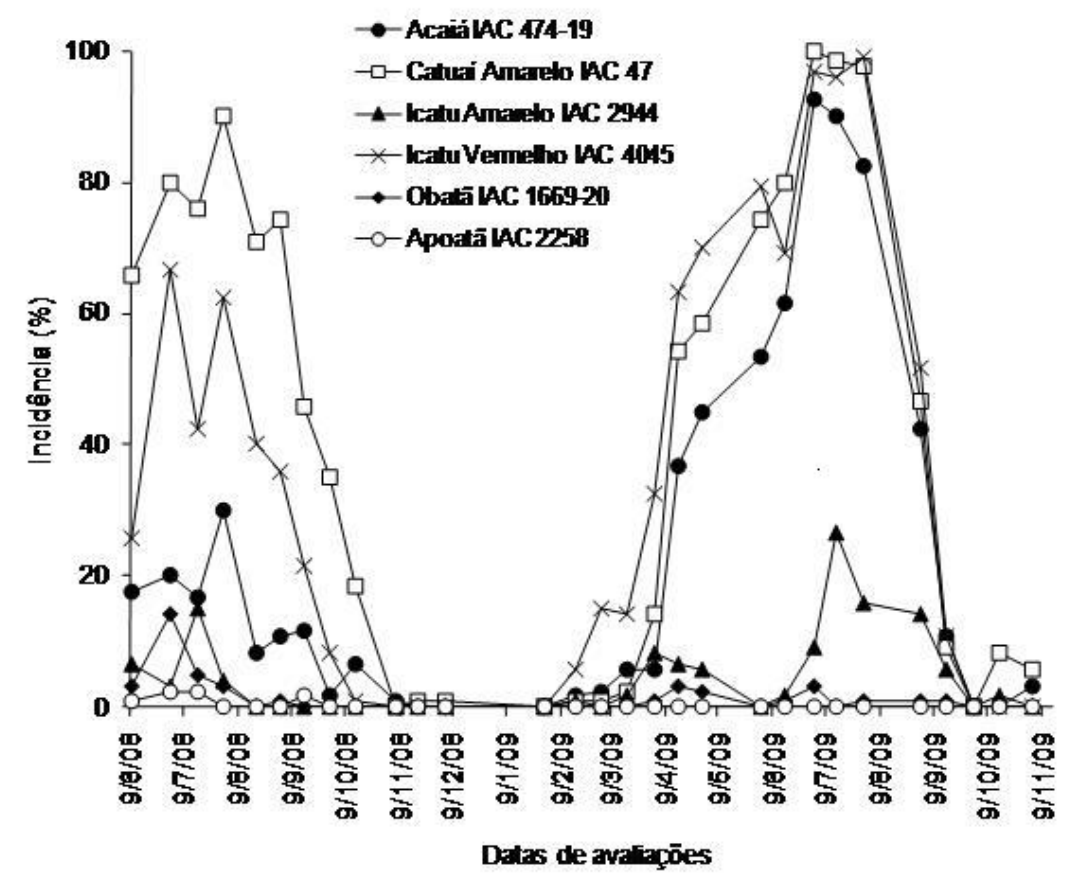

Fig. 1 - Curva do progresso da incidência da ferrugem alaranjada em cultivares de cafeeiro. Adamantina, SP, $2008-2009$.

Na prática tem-se com frequência a adoção de medidas de controle da ferrugem quando as lavouras apresentam 5 a $20 \%$ da doença (CunHA et al., 2004). A incidência da doença nos cafeeiros está representada na Figura 1.

Os cultivares Apoatã e a Obatã apresentaram incidência da ferrugem menor que 5\% em todo o período do estudo, excetuando-se a avaliação de 1/7/2008 quando se observou incidência de 14,2\% na Obatã. Merece esclarecer que os sintomas identificados como ferrugem nesses cultivares foram pequenas pontuações cloróticas, que corresponderiam ao início da incidência, mas que não evoluíram para a formação de uredósporos.

O Icatu Amarelo IAC 2944 de agosto/2008 até março/2009, durante cerca de 230 dias, mostrou incidência da doença menor que $5 \%$.

O Acaiá IAC 474-19 e o Catuaí Amarelo IAC 47, com comportamento similar, durante cerca de 150 dias, de novembro/08 até março/2009, não ultrapassaram o nível de $5 \%$ de incidência, o que também aconteceu com o Icatu Vermelho IAC 4045, de outubro/2008 a janeiro/2009, por um período de cerca de 110 dias.

A ocorrência da doença no cultivar Acaiá não foi baixa em 2008, pois, de julho a outubro, variou entre 10 e $30 \%$ de incidência, mas menos agressiva que em 2009, o que pode estar relacionado às possíveis diferenças no estado nutricional deste cultivar entre os anos do estudo.

A maior incidência da ferrugem no período experimental ocorreu em julho-agosto, sendo que o Catuai Amarelo, o Icatu Vermelho IAC 4045 e o
Acaiá IAC 474-19, em 2009, mostraram mais que 90\% das folhas infectadas com uredósporos, momento no qual o Icatu Amarelo IAC 2944 apresentava 26\% de incidência da doença.

No período entre outubro de 2008 e março de 2009, a incidência da ferrugem nos cultivares suscetíveis foi menor que 5\% (Fig. 1), exceção feita ao Icatu Vermelho IAC 4045, quando as temperaturas médias do ar foram maiores que $25^{\circ} \mathrm{C}$ e menores que $28,5^{\circ} \mathrm{C}$ e houve precipitação pluvial (Tabela 1). Nota-se que, embora a temperatura média do ar desse período seja considerada favorável ao desenvolvimento da doença (ZАмвоLIm et al., 2005), as temperaturas máximas médias mensais oscilaram entre $30^{\circ} \mathrm{C}$ e $33^{\circ} \mathrm{C}$ e ocorreram temperaturas absolutas máximas maiores que $34^{\circ} \mathrm{C}$ até $38^{\circ} \mathrm{C}$ (Tabela 1) que podem explicar os baixos valores de incidência constatados.

Relata-se que altas temperaturas impedem o desenvolvimento normal de $H$. vastatrix e são letais, mesmo quando o micélio já está estabelecido no interior da lâmina foliar, desde que a exposição à temperatura elevada seja suficientemente longa (Ribeiro et al., 1978). O período de incubação da ferrugem em cafeeiros a pleno sol foi maior quando as plantas foram submetidas à temperatura do ar de $31^{\circ} \mathrm{C}$, medidas em abrigo micrometeorológico, comparativamente àquelas mantidas à sombra, onde as temperaturas do ar não atingiam aquele valor (MORAEs et al., 1976). A exposição de cafeeiros a temperaturas próximas a $40^{\circ} \mathrm{C}$ por 4 horas durante 4 dias foi suficiente para impedir a evolução da moléstia (Ribeiro et al., 1978), 
Tabela 1 - Temperatura e precipitação pluvial dos anos da experimentação. Adamantina, SP, 2008-2009. Fonte CIIAGRO.

\begin{tabular}{|c|c|c|c|c|c|c|}
\hline \multirow{2}{*}{ Ano } & \multicolumn{2}{|c|}{ Temperatura absoluta } & \multicolumn{2}{|c|}{ Temperatura média mensal } & \multirow{2}{*}{$\begin{array}{c}\text { Temperatura } \\
\text { absoluta }\end{array}$} & \multirow{2}{*}{ Precipitação } \\
\hline & Máxima & Mínima & Máxima & Mínima & & \\
\hline 2008 & & & $-\left({ }^{\circ} \mathrm{C}\right)$ & & & $\mathrm{mm}$ \\
\hline junho & 31,0 & 5,0 & 26,6 & 13,2 & 19,9 & 35,6 \\
\hline julho & 32,2 & 11,0 & 29,1 & 14,0 & 21,6 & 0,2 \\
\hline agosto & 34,2 & 10,5 & 29,8 & 15,7 & 22,8 & 56,0 \\
\hline setembro & 36,8 & 8,7 & 29,3 & 14,2 & 21,7 & 29,9 \\
\hline outubro & 38,6 & 13,7 & 31,8 & 18,9 & 25,4 & 72,6 \\
\hline novembro & 34,7 & 15,5 & 32 & 18,9 & 25,4 & 45,4 \\
\hline dezembro & 38,0 & 13,3 & 32,9 & 20,0 & 26,5 & 143,0 \\
\hline 2009 & & & $-\left({ }^{\circ} \mathrm{C}\right)$ & & & $\mathrm{mm}$ \\
\hline janeiro & 35,8 & 17,3 & 30,2 & 20,3 & 25,3 & 403,8 \\
\hline fevereiro & 35,0 & 17,4 & 31,6 & 20,8 & 26,2 & 130,0 \\
\hline março & 35,5 & 16,6 & 31,7 & 19,9 & 25,8 & 116,0 \\
\hline abril & 32,9 & 13,7 & 31,0 & 17,1 & 24,1 & 1,8 \\
\hline maio & 32,3 & 7,6 & 28,7 & 15,6 & 22,2 & 57,7 \\
\hline junho & 29,0 & 3,1 & 24,3 & 11,6 & 17,9 & 28,9 \\
\hline julho & 31,6 & 4,6 & 25,6 & 13,9 & 19,8 & 73,9 \\
\hline agosto & 33,3 & 6,5 & 27,2 & 14,5 & 20,9 & 125,8 \\
\hline setembro & 34,2 & 13,1 & 29,4 & 18,0 & 23,8 & 117,4 \\
\hline outubro & 34,5 & 12,9 & 29,7 & 18,1 & 23,9 & 141,1 \\
\hline novembro & 38,0 & 20,7 & 33,0 & 22,1 & 27,5 & 115,4 \\
\hline dezembro & 34,8 & 18,3 & 31,0 & 20,9 & 25,9 & 407,7 \\
\hline
\end{tabular}

Merece atenção que de julho a setembro de 2008, quando se observou as maiores porcentagens de incidência do ano nos cafeeiros (Fig. 1), ocorreram temperaturas máximas médias mensais entre $29^{\circ} \mathrm{Ce}$ $30^{\circ} \mathrm{C}$ (Tabela 1) que, portanto, não foram suficientes para prejudicar a germinação dos uredósporos.

A incidência da ferrugem nos cultivares Acaiá IAC 474-19, Catuaí Amarelo IAC 47, Icatu Amarelo IAC 2944 e Icatu Vermelho IAC 4045 correlacionou-se significativa e negativamente com as temperaturas máxima e mínima e, no Obatã com a temperatura mínima (Tabela 2). A incidência de ferrugem nos cultivares não se correlacionou com a precipitação pluvial(Tabela 2). Estes resultados permitem concluir que o índice de incidência da ferrugem diminuiu com o aumento da temperatura, mas não com a precipitação pluvial.

As curvas de progresso da ferrugem obtidas para os cultivares Acaiá IAC 474-19, Catuaí Amarelo IAC 47 eIcatu Vermelho IAC 4045 (Fig. 1) assemelham-seà descrita para a maioria das regiões produtoras de café do Brasil,cujos índices de incidência maiores que $60 \%$ constatados em cafeeiros suscetíveis são relatados para cafezais com médio-alta produção (ZAMBOLIM et al., 2005). Os resultados obtidos assemelham-se também aos de Campinas, em que a porcentagem de folhas com ferrugem no cultivar Ouro Verde Amarelo IAC 4397 foi superior a $80 \%$ no período de julho a setembro de 2003 e caiu drasticamente até zero em outubro (CONCEIÇÃo, 2005).

As menores porcentagens de incidência da ferrugem, na média dos 2 anos do estudo, se observaram nos cultivares Apoatã, Obatã e Icatu Amarelo
IAC 2944 (Fig. 1), o que resultou em menor $(\mathrm{P}<$ $0,05)$ área abaixo da curva de progresso da doença comparativamente à obtida para o Acaiá, o qual diferiu daquelas áreas definidas pelas curvas com os índices de incidência dos cultivares Icatu Vermelho IAC 4045 e Catuaí Amarelo IAC 47 (Tabela 3).

O Apoatã IAC 2258 (FAzUOLI, 1999), o Obatã IAC 1669-20, o Icatu Amarelo 2944 e o Icatu Vermelho IAC 4045 são cultivares de cafeeiro para os quais a presença de resistência (Aguiar et al., 2004; THOMAZIELO et al., 2000; FAZUOLI, 1999) ou resistência tolerância (DiAs et al., 2005) à ferrugem alaranjada é relatada, enquanto o Acaiá IAC 474-19 e o Catuaí Amarelo IAC 47 são descritos como suscetíveis (FAzUoli, 1999; ThOMAZielo et al., 2000; Aguiar et al., 2004; Dias et al., 2005).

O desenvolvimento da ferrugem alaranjada no Icatu Amarelo IAC 2944, em 2009, eno Icatu Vermelho IAC 4045 (Fig. 1), descritos como resistentes à doença (FAZUoli, 1999; Thomazielo et al., 2000; Aguiar et al., 2004), pode ser decorrente da presença de uma ou mais raças de $H$. vastatrix no local da experimentação e às quais não se submeteram os cultivares durante o processo de seleção. A presença de novas raças fisiológicas do fungo nas plantações do Brasil é sugerida pela constatação de esporos da ferrugem em cultivares anteriormente considerados resistentes à doença, como o Icatu, Catucaí e derivados do Híbrido de Timor (FAzUoli et al., 2007). O resultado é importante, pois, como no Obatã IAC 1669-20, o controle químico da ferrugem não é recomendado nos cultivares Icatu Amarelo IAC 2944 e Icatu Vermelho IAC 4045 (THOMAZIELO, 2000). 
Tabela 2 - Coeficientes de correlação (r) entre incidência da ferrugem em cultivares de cafeeiro e dados climáticos de precipitação pluvial e temperatura de Adamantina, SP, entre 6/2008 e 11/2009 (30 avaliações).

\begin{tabular}{lccc}
\hline Cultivar & $\begin{array}{c}\text { Ferrugem/Precipitação } \\
\text { acumulada }^{1}\end{array}$ & $\begin{array}{c}\text { Ferrugem/Temperatura } \\
\text { máxima }^{2}\end{array}$ & $\begin{array}{c}\text { Ferrugem/Temperatura } \\
\text { mínima }^{2}\end{array}$ \\
\hline Acaiá & $-0,21 \mathrm{~ns}$ & $-0,72^{*}$ & $-0,63^{*}$ \\
Catuaí Amarelo & $-0,35 \mathrm{~ns}$ & $-0,72^{*}$ & $-0,85^{*}$ \\
Icatu Amarelo & $-0,12 \mathrm{~ns}$ & $-0,54^{*}$ & $-0,40^{*}$ \\
Icatu Vermelho & $-0,30 \mathrm{~ns}$ & $-0,69^{*}$ & $-0,70^{*}$ \\
Obatã & $-0,21 \mathrm{~ns}$ & $-0,29 \mathrm{~ns}$ & $-0,41^{*}$ \\
Robusta & $-0,16 \mathrm{~ns}$ & $-0,20 \mathrm{~ns}$ & $-0,33 \mathrm{~ns}$ \\
\hline
\end{tabular}

*Significativo pelo teste $\mathrm{F}(\mathrm{P}<0,05)$.

${ }^{1}$ Precipitação acumulada em cada período de avaliação.

${ }^{2}$ Média da temperatura em cada período de avaliação.

Tabela 3 - Valores médios da área abaixo da curva de progresso doença (AACPD) ferrugem alaranjada em cultivares de cafeeiro. Adamantina, SP, 2008-2009 (30 avaliações).

\begin{tabular}{lccc}
\hline \multirow{2}{*}{ Cultivar } & & AACPD $^{1}$ & \\
\cline { 2 - 4 } & 2008 & 2009 & Total \\
\hline Apoatã IAC 2258 & $35,3 \mathrm{~A}$ & $0,0 \mathrm{~A}$ & $35,3 \mathrm{~A}$ \\
Obatã IAC 1669-20 & $134,1 \mathrm{~A}$ & $62,4 \mathrm{~A}$ & $196,5 \mathrm{~A}$ \\
Icatu Amarelo IAC 2944 & $129,1 \mathrm{~A}$ & $550,1 \mathrm{~B}$ & $679,3 \mathrm{~A}$ \\
Acaiá IAC 474-19 & $578,1 \mathrm{~B}$ & $3040,3 \mathrm{C}$ & $3618,4 \mathrm{~B}$ \\
Icatu Vermelho IAC 4045 & $1440,9 \mathrm{C}$ & $3885,5 \mathrm{C}$ & $5326,4 \mathrm{C}$ \\
Catuaí Amarelo IAC 47 & $2562,9 \mathrm{D}$ & $3597,4 \mathrm{C}$ & $6167,4 \mathrm{C}$ \\
\hline C.V.(\%) & 15,9 & 12,4 & 18,0 \\
\hline
\end{tabular}

\section{REFERÊNCIAS}

AGUIAR, A.T.F.; GUERREIRO FILHO, O.; MALUF, M.P., GALLO, P.B.; FAZUOLI, L.C. Caracterização de cultivares de Coffea arábica mediante a utilização de descritores mínimos. Bragantia, v. 63, p.179-192, 2004.

CAMPBELL, C.L.; MADDEN, L.V. Introduction to plant disease epidemiology. New York NY: John Wiley, 1990. $532 \mathrm{p}$.

CHALFOUN, S.M. Doenças do cafeeiro: importância, identificação e métodos de controle. Lavras: UFLA/ FAEPE, 1997. 96p.

CONCEIÇÃO, C.H. Biologia, dano e controle do bicho-mineiro em cultivares de café arábica. 2005. 86f. Dissertação (Mestrado - Agricultura Tropical e Subtropical) - Instituto Agronômico, Campinas, 2005.

CUNHA, R.L.; MENDES, A.N.G.; CHALFOUN, S.M. Controle químico da ferrugem do cafeeiro (Coffea arabica L.) e seus efeitos na produção e preservação de enfolhamento. Ciência e Agrotecnologia, v. 28, n.5, p.990-996, 2004.

DIAS, F.P.; SOUZA, C.A.S.; MENDES, A.N.G.; CARVALHO, S.P.N.; RASO, B.S.M,; BOTELHO, C.E. Caracterização de progênies de cafeeiro (Coffea arábica L.) selecionadas em Minas Gerais: II - caracteres relacionados à produção. Revista Ceres, v.52, n.299, p.85-100, 2005.
FAZUOLI, L.C. Cultivares IAC de café. O Agronômico, v.51, n.1, p.27-34, 1999.

FAZUOLI, L.C.; BRAGHINI, M.T.; SILVAROLLA, M.B., OLIVEIRA, A.C.B. A ferrugem alaranjada do cafeeiro e a obtenção de cultivares resistentes. O Agronômico, v.59, n.1, p.48-53, 2007.

GALLI, F.; CARVALHO, P.C.T. Doenças do cafeeiro. In: GALLI, F. (Coord.). Manual de fitopatologia. São Paulo: Editora Ceres, 1980.587p. V.2. p.128-140.

GARÇON, C.L.P.; ZAMBOLIM, L.; MIZUBUTI, E.S.G.; VALE, F.X.R.; COSTA, H. Controle da ferrugem do cafeeiro com base no valor de severidade. Fitopatologia Brasileira, v.29, n.5, p.486-549, 2004.

MIGUEL, A.E.; MATIELLO, J.B.; MANSK, Z.; ALMEIDA, S.R. Observações sobre os efeitos de três níveis de produção na incidência e controle da ferrugem do cafeeiro. In: CONGRESSO BRASILEIRO DE PESQUISAS CAFEEIRAS, 5., 1977, Guarapari, ES. Resumos. Rio de Janeiro: 1977. p.220-221.

MORAES, A.S.; SUGIMORI, M.H.; RIBEIRO, I.J.A.; ORTOLANI, A.A; PEDRO JUNIOR, M. Período de incubação de Hemileia vastatrix Berk et Br. em três regiões do Estado de São Paulo. Summa Phytopathologica, v.2, n.1, p.32-38, 1976.

RIBEIRO, I.J.A.; MÔNACO, L.C., TISSELLI FILHO, O.; SUGIMORI, M.H. Efeito da temperatura no desenvol- 
vimento de Hemileia vastatrix em cafeeiro suscetível. Bragantia, v.37, n.2, p.11-16, 1978.

THOMAZIELO, R.A.; FAZUOLI, L.C.; PEZZOPANE JUNIOR, M.P.; FAHL, J.I., CARELL, M.L.C. Café arábica: culturas e técnicas de produção. Campinas: Instituto Agronômico, 2000. 82p. (Boletim Técnico, 187).

TREMOCOLDI, W.A.; BRUNINI, O. Caracterização agroclimática das unidades da Secretaria de Agricultura e Abastecimento do Estado de São Paulo: Adamantina e Região. Campinas: Instituto Agronômico, 2008. 37p. (Série Tecnologia APTA, Boletim Técnico IAC, 204).

VIEIRA JUNIOR, J.R.; FERNANDES, C.F.; RODRIGUES, V.G.S.; BENTES-GAMA, M.M.; SILVA, D.S.G.; FERNANDES, S.R.; DIOCLESIANO, J.M. Avaliação da severidade da ferrugem (Hemileia vastatrix) em cafeeiros (Coffea canephora) cultivados em condições de sombreamento. Porto Velho: Embrapa, 2008. 4p. (Circular Técnica 103).

ZAMBOLIM, L.; VALE, F.X.R.; PEREIRA, A.A.; CHAVES, G.M. Café (Coffea arábica L.): Controle de doenças causadas por fungos, bactérias e vírus. In: VALE, F.X.R.; ZAMBOLIM, L. (Ed.). Controle de doenças de plantas. Viçosa: Suprema Gráfica e Editora, 1997. p.83-180.

ZAMBOLIM, L.; VALE, F.X.R.; ZAMBOLIM, E.M.

Doenças do cafeeiro (Coffea arábica e Coffea canephora). In: KIMATI, H.; AMORIM, L.; REZENDE, J.A.M.; BERGAMIM FILHO, A.; CAMARGO, L.E.A. (Ed.). Manual de fitopatologia: doenças das plantas cultivadas. 4.ed. São Paulo: Agronômica Ceres, 2005. 663p.

Recebido em $1 / 8 / 11$

Aceito em 29/10/12 